\title{
RELATIVE EXTREMA OF PAIRS OF QUADRATIC AND HERMITIAN FORMS*
}

BY

\author{
R. G. D. RICHARDSON
}

INTRODUCTION

The problem of relative extrema of pairs of forms, which plays an increasingly important rôle in analysis, concerns two quadratic forms

$$
A(x, x) \equiv \sum_{i j}^{1, n} a_{i j} x_{j} x_{i}, \quad B(x, x) \equiv \sum_{i j}^{1, n} b_{i j} x_{j} x_{i} ; a_{i j}=a_{j i}, b_{i j}=b_{j i},
$$

or two hermitian forms

$$
A(z, \bar{z})=\sum_{i j}^{1, n} a_{i j} z_{j} \bar{z}_{i}, \quad B(z, \bar{z})=\sum_{i j}^{1, n} b_{i j} z_{i} \bar{z}_{j} ; a_{i j}=\bar{a}_{j i}, b_{i j}=\bar{b}_{j i}
$$

Does the form $A$ possess a minimum for those real values of the $x$ 's or complex values of the $z$ 's which fulfil the condition $B=1$ and perhaps in addition certain auxiliary linear conditions; and if so, how is it obtained?

In the Introduction to the preceding memoir,t the author has indicated the intimate connection between this problem and that of equivalence of pairs of forms. Following the usual method of the Lagrange factor as outlined there for the problem

$$
A(x, x)=\text { Min., } \quad B(x, x)=1,
$$

we consider the extrema of the form $A+\lambda B$, which leads on differentiation to the set of necessary conditions

$$
\sum_{j}\left(a_{i j}-\lambda b_{i j}\right) x_{j} \quad(i=1,2, \cdots, n)
$$

A necessary and sufficient condition that a solution of these equations exist is that $\lambda$ be one of the real roots $\lambda_{1} \leq \cdots \leqq \lambda_{n}$, called characteristic

* Presented to the Society, September 7, 1923.

† $A$ new method in the equivalence of pairs of bilinear forms, these Transactions, vol. 26, pp. 451-478. This will be referred to as I. 
numbers, of the determinant $\left|a_{i j}-\lambda b_{i j}\right|$. Denoting a solution corresponding to $\lambda_{k}$ by

$$
X^{(k)}=\left(x_{1}^{(k)}, \cdots, x_{n}^{(k)}\right)
$$

and multiplying the equations (2) by $x_{1}^{(k)}, \ldots, x_{n}^{(k)}$, respectively, and adding, we obtain

$$
A\left(X^{(k)}, X^{(k)}\right)-\lambda_{k} B\left(X^{(k)}, X^{(k)}\right)=0
$$

a relation which all solutions must satisfy. It follows from (3) that the extremal value of the problem must be sought among the numbers $\lambda_{i}$ and in this case it is $\lambda_{1}$.

In the case of the problem with hermitian forms

$$
A(z, \bar{z})=\text { Min., } \quad B(z, \bar{z})=1,
$$

the usual method of differentiating with regard to $\bar{z}_{i}$ the expression $A+\lambda B$ gives

$$
\sum_{j}\left(a_{i j}+\lambda b_{i j}\right) z_{j}=0,
$$

while differentiation with regard to $z_{i}$ gives a similar set, which, being the conjugates of $\left(2^{\prime}\right)$, add nothing new. On multiplying equations $\left(2^{\prime}\right)$ respectively by $\bar{z}_{1}^{(k)}, \ldots, \bar{z}_{n}^{(k)}$ and adding, there results

$$
A\left(Z^{(k)}, \bar{Z}^{(k)}\right)-\lambda_{k} B\left(Z^{(k)}, \bar{Z}^{(k)}\right) .
$$

Returning now to the minimum problem (1) and adding the orthogonal condition

$$
B\left(X^{(1)}, x\right) \equiv \sum_{i j}^{1, n} b_{i j} x_{j}^{(1)} x_{i}=0,
$$

we are led to consider the minimum of the expression

$$
A(x, x)-\lambda B(x, x)-2 \mu B\left(X^{(1)}, x\right),
$$

which on differentiation with regard to $x_{i}$ gives the set of non-homogeneous linear equations

$$
\sum_{j}\left(a_{i j}-\lambda b_{i j}\right) x_{j}=\mu \sum_{j} b_{i j} x_{j}^{(1)} .
$$


If $B$ is definite, we shall see $(\S 2)$ that $\mu=0$; hence the solution of the extended problem is to be found among the solutions of (2) and the minimal value is $\lambda_{z}$ furnished by $X^{(2)}$. Further linear orthogonal conditions may be successively added, the formal expression to be minimized in the last problem being

$$
A(x, x)-\lambda B(x, x)-2 \mu_{1} B\left(X^{(1)}, x\right)-\cdots-2 \mu_{n-1} B\left(X^{(n-1)}, x\right) .
$$

It turns out again that in case $B$ is a definite form (or even more generally), the $\mu$ 's are all zero, and hence the solutions of the extended problems are contained among the solutions of (2), being $X^{(3)}, \ldots, X^{(n)}$ respectively and giving on account of (3) the minima $\lambda_{3}, \ldots, \lambda_{n}$.

While it is true that in such minimum problems actual extrema exist only in the special cases where the forms are real quadratic or hermitian and one of them definite $(\S 1)$, the formal work can be carried out, so far as the first derivatives (such as (2) and (4) and a corresponding set of equations in $y$ ) are concerned, in the most general case of bilinear forms with complex coefficients. Besides introducing new results we have in the previous paper ventured to hope that such a point of view may give a unity to the treatment of the problem of equivalence. Let us for a moment consider how the discussion of the extrema must be modified to include other cases.

In the irregular cases where $B(x, x)$ is not definite, $B\left(X^{(1)}, X^{(1)}\right)$ may vanish and in order to make it unity, the corresponding values of $x$ must be infinite. In order that the minimum problem (1) have a meaning even formally, it would then be necessary to modify it and to write

$$
A(x, x)=\text { Min. } \quad B(x, x)=0 .
$$

Further, when $B(x, x)$ is not definite, it may turn out that in the equations such as (4) the multiplier $\mu$ is not zero and that these equations do not reduce to (2). The formal extremum in this case is then not a solution of the equation (2); we have seen in I ( $\$ 5)$ that corresponding to this phenomenon is the fact on the algebraic side that the equations (2) have less than $n$ linearly independent solutions. In this event $\mu$ may be given the value unity (that is, absorbed into the solution $X^{(1)}$ ), and it was there shown that the solutions are the same as those of the equations obtained from (2) by differentiation with regard to $\lambda$, the sets of constants $X_{i}^{(1)}$ being regarded as solutions of the original equation. The discussion was made to cover further extensions of the problem. If the $\mu$ 's are not all zero, we saw that derived equations such as are suggested by the 
formal minimum problem and their solutions played a fundamental rôle in the problems of equivalence.

The literature of relative extrema of quadratic and hermitian forms contains no set of sufficient conditions. In the general theory of extrema without auxiliary conditions, the criterion associated with the second derivatives asserts* that if the sign of a determinant formed from these derivatives and the signs of certain of its minors are all positive for those values of the variables which make the first derivatives vanish, a minimum is present; if the determinant is negative and the minors are alternately positive and negative for these values, a maximum is present; if the determinant vanishes, no test is furnished. Unfortunately even in the simplest case of relative extrema, a corresponding test fails because of the vanishing of this crucial determinant of the second derivatives. This we shall now proceed to demonstrate.

Denoting by $A$ and $B$ two functions of $n$ variables such that $B$ is definite we can calculate the second derivatives of the quotient $A / B$ for those values $\left(x_{1}\right)_{0}, \cdots,\left(x_{n}\right)_{0}$ which make the first derivatives zero. These are

$$
\left.\left.\frac{\partial^{2}(A / B)}{\partial x_{i} \partial x_{k}}\right]_{0}=\frac{B \frac{\partial^{2} A}{\partial x_{i} \partial x_{k}}-A \frac{\partial^{2} B}{\partial x_{i} \partial x_{k}}}{B^{2}}\right]_{0}
$$

For the particular case of relative extrema of quadratic or hermitian forms, $B$ can be set equal to unity while for those values of the'variables which are obtained by setting the first derivatives equal to zero we have seen that $A=\lambda_{k}$, where $\lambda_{k}$ is a zero of the determinant $\left|a_{i j}-\lambda b_{i j}\right|$. Since

$$
\frac{\partial^{2} A}{\partial x_{i} \partial x_{i}}=2 a_{i k}
$$

etc., we find then that the combination of second derivatives to be scrutinized is, except for a constant factor $2^{n}$, the determinant $\left|a_{i j}-\lambda_{k} b_{i j}\right|$ which vanishes for all such $\lambda_{k}$ and hence furnishes no criterion.

It is possible, however, by a different method of attack to deduce sufficient conditions for relative extrema. A new necessary condition is first developed in $\S 3$ and this turns out to be incisive enough to complete the set of criteria for sufficiency.

\section{ExTREMa of QUADRATIC Forms}

In discussing the relative extrema of a pair of quadratic forms, it is necessary to confine attention to the case where the coefficients of both

\footnotetext{
* Hancock, Theory of Maxima and Minima, pp. 91-92.
} 
are real. Unless the contrary is stated, only non-singular quadratic forms will come under consideration, and they will be denoted by $Q_{1}(x, x), Q_{2}(x, x)$ instead of $A(x, x), B(x, x)$. The simplest problem arises when one of the forms is definite. Let us then consider first those real values of the variables $x_{i}$ which give to the positive definite form $Q_{2}(x, x)$ the value 1 . For these limited values the form $Q_{1}(x, x)$ can take on limited values only, and there will be extrema which can be obtained in the usual way. If $Q_{1}$ also is definite, the extrema will have the same sign; but if indefinite, the minimum will be negative and the maximum positive.

If $Q_{1}$ is positive definite and we set it equal to 1 , the form $Q_{2}$ will have for extrema the reciprocals of the extrema obtained in the original problem; if it is negative definite and is set equal to -1, the extrema of $Q_{2}$ will have the absolute values of these reciprocals. If on the other hand $Q_{1}$ is indefinite and it is set equal to 1 , the minimum of $Q_{2}$ will be the reciprocal of the extremum of the other problem, but the maximum will be infinite, while for those values which make $Q_{1}=-1$, the minimum of $Q_{2}$ will be the absolute value of a reciprocal of an extremum of the other problem and the maximum will be infinite.

The ratio $Q_{1} / Q_{2}$ is, of course, the fundamental point for consideration and when $Q_{2}$ is indefinite and hence becomes zero for values of the variables other than zero, the ratio can be made infinite unless the two forms are linearly dependent. When singular forms are allowed to enter into the discussion it may be that, for the infinite values of the $x$ 's which are permitted, there may be a finite extremum. For example, the singular form $Q_{1} \equiv\left(x_{1}+x_{2}\right)^{2}$ has a minimum zero for those infinite values of the variable which make $Q_{2} \equiv x_{1}^{2}-x_{2}^{2}$ equal to 1 . But in any case a positive definite form must have a finite minimum, a negative must have a finite maximum.*

To get a somewhat different point of view, let us consider the extremes as functions of the coefficients of $Q_{1}, Q_{2}$. If at the beginning both forms are positive definite and $Q_{2}$ is set equal to unity, one can continuously alter the coefficients of $Q_{2}$ so that it passes over into an indefinite form. The maximum of $Q_{1}$ increases without limit, the minimum remains finite. When $Q_{2}$ during the transition becomes singular-definite, the maximum of $Q_{1}$ is infinite and remains so when the form becomes indefinite. But when $Q_{\mathbf{z}}$ is indefinite it may also be equated to -1 and a corresponding maximum of infinity and a finite minimum for $Q_{1}$ will result.

* Let $Q_{1}$ be an indefinite form and $Q_{2}$ a singular definite form. If $Q_{2}$ is positive its minimum will be zero and this will occur for values which make $Q_{1}=1$ or $Q_{1}=-1$ or for both (e. g., if $Q_{2}=x_{1}^{2}+x_{2}^{2}$ and (1) $Q_{1}=x_{1}^{2}-x_{2}^{2}+x_{3}^{2}$; (2) $Q_{1}=x_{1}^{2}-x_{2}^{2}-x_{3}^{2}$; (3) $Q_{1}=x_{1}^{2}-x_{2}^{2}-x_{8}^{2}+x_{4}^{2}$ ). The minimum of $Q_{2}$ for $Q_{1}=1$, or for $Q_{1}=-1$ may, however, be greater than zero (e. g. in (2)). 
If, however, initially the form $Q_{1}$ is indefinite and $Q_{2}$ passes over from positive to indefinite, the situation is somewhat different. Consider, for example, the forms

$Q_{1} \equiv x_{1}^{2}+x_{2}^{2}-x_{3}^{2}-x_{4}^{2}, \quad Q_{2}=x_{1}^{2}+\varepsilon_{2} x_{2}^{2}+\varepsilon_{3} x_{3}^{2}+x_{4}^{2}=1, \quad \varepsilon_{2}, \varepsilon_{3}>0$.

If $\varepsilon_{2}$ approaches zero, $Q_{1}$ has a maximum approaching $+\infty$ and a finite minimum, while as $\varepsilon_{3}$ approaches zero, $Q_{1}$ has a minimum approaching - $\infty$ and a finite maximum. When $\varepsilon_{2}$ or $\varepsilon_{3}$ changes sign, $Q_{2}$ may be set equal to -1 and new extrema of $Q_{1}$ enter. It is not difficult to see that various cases will arise in problems of this type according as $Q_{1}+k Q_{2}$ is or is not a definite form for some value of $k$. We shall proceed now to develop the general theory.

TheоRem I. If for values of the variables which give to the non-singular quadratic form $Q_{2}$ the value $c \neq 0$ the form $Q_{1}$ is positive (negative) and has a minimum (maximum) zero, then $Q_{1}$ is a singular positive (negative) definite form.

For, let $Q_{1}$ be reduced by a real non-singular transformation to the form $Q_{1}=\sum_{i} \varepsilon_{i} x_{i}^{2}$, where some of the $\varepsilon$ 's may be positive and some negative. $Q_{2}$ will by the same transformation take on a new form. If we differentiate the quotient $Q_{1} / Q_{2}$ with regard to each of the variables, and note that by hypothesis for the minimum point in question the numerator is zero and that these derivatives are all zero and $Q_{2}=c$, we get the equations $\varepsilon_{i} x_{i}=0(i=1, \cdots, n)$. Since not all the $x_{i}$ can vanish, it follows that at least one of the $\varepsilon^{\prime}$ s (say $\varepsilon_{1}$ ) is zero. The form $Q_{1}$ is then singular. It is possible to show further that $Q_{1}$ is definite, for example that none of the $\varepsilon$ 's are negative. In order to do this let it be assumed on the contrary that $\varepsilon_{2}=-1$. Two cases must be considered according as the corresponding values of $x_{1}$ in $Q_{2}$ and of any other variables which occur in $Q_{2}$ but not in $Q_{1}$ are finite or infinite. When this is finite, we note that it is possible to chose it slightly larger, thus making $Q_{1}$ negative and giving to $Q_{\mathrm{z}}$ the value $c+\varepsilon$ which has the same sign as $c$. By multiplying all the variables by $\sqrt{c /(c+\varepsilon)}$ the numerator remains negative and the denominator is $c$, which is a contradiction of the hypothesis.

If the case of infinite values is considered and $x_{1}$ is the only variable missing from $Q_{1}$, it cannot occur in $Q_{2}$ to a power higher than the first. For since the other values of the $x$ 's are finite, a square term would be an infinity of higher order than the others and would make $Q_{2}$ infinite. $Q_{2}$ may then be written $x_{1} L\left(x_{2}, \cdots, x_{n}\right)+Q^{\prime}\left(x_{2}, \cdots, x_{n}\right)$ where $L$ and $Q^{\prime}$ are respectively linear and quadratic forms in one less variable. But now $x_{2}, \cdots, x_{n}$ can be assigned in $Q_{1}$ and $Q_{2}$ making the former negative 
while $x_{1}$ can then be assigned to make $Q_{2}=c$, which contradicts the hypothesis.

Again if there were variables in $Q_{z}$ other than $x_{1}$ which did not occur in the numerator and which were infinite for the minimum, the quadratic form composed of these terms alone must be indefinite, for otherwise $Q_{2}$ would be infinite. These variables may then be adjusted to make $Q_{2}=c$ after the numerator is made negative, which leads to the same contradiction as above.

THEOREM II. If for values of the variables which give to the indefinite form $Q_{2}$ the value $c \neq 0$ the indefinite form $Q_{1}$ has a minimum (maximum) $k$, it may be written

$$
Q_{1}=\frac{k}{c} Q_{2}+P \quad\left(Q_{1}=\frac{k}{c} Q_{2}-P\right)
$$

where $P$ is a positive singular form.

This follows immediately from the preceding theorem since the minimum of $Q_{1}-(k / c) Q_{2}$ under the conditions imposed is zero.

COROLlary. When $Q_{1}$ and $Q_{2}$ are indefinite but there exist constants $k_{1}, k_{2}$ such that $k_{1} Q_{1}+k_{2} Q_{2}$ is singular definite, the ratio $Q_{1} / Q_{2}$ takes on the values 0 and $\pm \infty$ but there may be a single continuous range of values which it does not take on.

For, on writing

$$
\frac{k_{1} Q_{1}}{k_{2} Q_{2}}+1=\frac{P}{k_{2} Q_{2}}
$$

where $P$ is a singular definite positive form, we have (cf. footnote p. 483) that the expression on the right may for positive $k_{2} Q_{2}$ take on values from a positive constant (or zero) to $+\infty$ while for negative $k_{2} Q_{2}$ it takes on values from negative constant (or zero) to $-\infty$. If both ranges include zero the ratio can be any value whatever; but if not, there is a gap in the values taken on by $k_{1} Q_{1} / k_{2} Q_{2}$ and hence by $Q_{1} / Q_{2}$.

\section{RELATIVE MINIMA OF QUADRATIC AND HERMITIAN FORMS}

In taking up the formal analysis of the actual problems of relative extrema, we note from the discussion of the preceding section that for quadratic forms nothing is lost in generality by assuming that the form $B$ is positive definite and has the value unity $(B(x, x)=1)$. By means of a real substitution the pair of quadratic forms can be reduced in this case to

$$
A(x, x)=\sum \lambda_{i} x_{i}^{2} ; \quad B(x, x)=\sum x_{i}^{2}, \quad \lambda_{1} \leqq \lambda_{2} \leqq \cdots \leqq \lambda_{n} .
$$


The minimum $\lambda_{1}$ of $A$ for those values which make $B(x, x)=1$ is thus given by $x_{1}^{(1)}=1, x_{2}^{(1)}=\cdots=x_{n}^{(1)}=0$ and the maximum $\lambda_{n}$ by $x_{1}^{(n)}=$ $\cdots=x_{n-1}^{(n)}=0, x_{n}^{(n)}=1$. If in addition to the quadratic condition it is demanded that the solution be orthogonal to the minimum already found, $B\left(X^{(1)}, x\right)=0$, it is necessary that $x_{1}=0$ and the minimum $\lambda_{2}$ is furnished by $x_{2}^{(2)}=1, x_{1}^{(2)}=x_{3}^{(2)}=\cdots=x_{n}^{(2)}=0$. And in general, the solution $x_{1}^{(m)}=\cdots=x_{m-1}^{(m)}=x_{m+1}^{(m)}=\cdots=x_{n}^{(m)}=0, x_{m}^{(m)}=1$ gives $\lambda_{m}$ as the minimum of $A$ under the quadratic condition $B=1$ and the linear conditions of orthogonality, $B\left(X^{(k)}, x\right)=0$, when $k$ ranges over $1, \cdots, m-1$, and as the maximum under the quadratic and the same linear condition when $k$ ranges over $m+1, \ldots, n$.

These results may be immediately translated into terms of the original forms $A$ and $B$.

THEOREM III. If $A(x, x)$ and $B(x, x)$ are two real non-singular quadratic forms of which $B$ is positive definite and if $\lambda_{1} \leqq \lambda_{2} \leqq \cdots \leqq \lambda_{n}$ are the $n$ characteristic numbers of the $\lambda$-determinant $\left|a_{i j}-\lambda b_{i j}\right|$, then the minimum of $A(x, x)$ for which $B(x, x)=1$ is $\lambda_{1}$ furnished by $X^{(1)}$ and the maximum is $\lambda_{n}$ furnished by $X^{(n)}$; and in general $X^{(m)}$ furnishes the minimum $\lambda_{m}$ under the added conditions $B\left(X^{(1)}, x\right)=0, \cdots, B\left(X^{(m-1)}, x\right)=0$ and the maximum $\lambda_{m}$ under the added conditions $B\left(X^{(m+1)}, x\right)=0, \cdots$, $B\left(X^{(n)}, x\right)=0$.

This theorem may also be derived directly and the actual existence of the extrema shown by expansion* in terms of constants in somewhat the

* Since any set of $n$ constants $C_{1}, \ldots, C_{n}$ can be expressed linearly in terms of any $n$ given linearly independent sets of $n$ constants each, either set of normalized solutions in form like (16) or (16') of $I$ will serve as a basis for such expansion. To calculate the coefficients let us denote them by $c_{1}, \cdots, c_{n}$ and write

$$
c_{j}=c_{1} x_{j}^{(1)}+\cdots+c_{k} x_{j}^{(k)}+\cdots+c_{n} x_{j}^{(n)} .
$$

When $n$ corresponds to a solution of multiplicity unity and these equations are multiplied respectively by $b_{1 j} y_{1}^{(k)}+\cdots+b_{n j} y_{n}^{(k)}$ and the result summed over $j$, the first two theorems of $I$ determine the coefficient as $\Sigma_{i j} C_{j} b_{i j} y_{1}^{(k)}=c_{k}$. If on the other hand $n$ corresponds to a multiple solution and is one of the numbers $\mu+1, \mu+2, \cdots, \mu+m_{r}$ which correspond successively to a primary and its corresponding solution of the derived equations, it is necessary to multiply by

$$
b_{i j} y_{1}^{\left(2 \mu+m_{r}-k+1\right)}+\cdots+b_{i j} y_{n}^{\left(2 \mu+m_{r}-k+1\right)}
$$

and in that case from the theorems of $I(\S 5)$ we have, by a similar process,

$$
\sum_{i j} C_{j} b_{i j} y_{i}^{\left(2 \mu+m_{r}-k+1\right)}=c_{k} \text {. }
$$

If the $y$ 's are the same as the $x$ 's, the results are correspondingly modified. 
same fashion as was done in an article in these Transactions.* But the process ordinarily used in analysis is more closely allied to the methods indicated in the Introduction and we shall proceed in that direction. We propose therefore to treat in detail by the ordinary methods of relative maxima and minima some one problem, and have chosen for this purpose hermitian forms, which are more general than the real quadratic.

In order that a hermitian form $H(z, \bar{z})$ be definite it is necessary and sufficient that the quadratic form in the $x$ 's and $y$ 's into which it may be resolved by the substitution $z=x+i y$ be definite also. In discussing the relative extrema we shall then assume that $B(z, \bar{z})$ is positive definite. Since the problem of maxima is treated in the same fashion, only the minima will be discussed. The simplest problem may be set up as follows:

$$
A(z, \bar{z})=\text { Min., } \quad B(z, \bar{z})-1=0 .
$$

Proceeding in the manner usual in the analogous problem for real variables where the variables and coefficients are real, let us consider the expression

$$
A(z, \bar{z})-\lambda[B(z, \bar{z})-1]=\operatorname{Min} .
$$

When $\lambda$ is real, this real hermitian form can be derived with regard to each $x$ and each $y$, the $2 n$ partial derivatives being real expressions to be equated to zero to furnish the minimum. It is, however, not necessary to resort to that device. If we derive with regard to $z_{i}$ there result $n$ expressions to be equated to zero, deriving with regard to $\bar{z}_{i}$ giving expressions conjugate to the first set which on equating to zero give nothing new.

From the relations

and

$$
x_{i}=\frac{z_{i}+\bar{z}_{i}}{2}, \quad y_{i}=\frac{z_{i}-\bar{z}_{i}}{2 \sqrt{-1}}
$$

$$
\frac{\partial H}{\partial z_{i}}=\frac{\partial H}{\partial x_{i}} \cdot \frac{\partial x_{i}}{\partial z_{i}}+\frac{\partial H}{\partial y_{i}} \cdot \frac{\partial y_{i}}{\partial z_{i}}, \quad \frac{\partial H}{\partial \bar{z}_{i}}=\frac{\partial H}{\partial x_{i}} \cdot \frac{\partial x_{i}}{\partial \bar{z}_{i}}+\frac{\partial H}{\partial y_{i}} \cdot \frac{\partial y_{i}}{\partial \bar{z}_{i}}
$$

it follows that

$$
\frac{\partial H}{\partial z_{i}}=\frac{1}{2} \frac{\partial H}{\partial x_{i}}-\frac{1}{2} \frac{\partial H}{\partial y_{i}} V \overline{-1}, \quad \frac{\partial H}{\partial z_{i}}=\frac{1}{2} \frac{\partial H}{\partial x_{i}}+\frac{1}{2} \frac{\partial H}{\partial y_{i}} V \overline{-1} .
$$

Since the expressions $\partial H / \partial x_{i}$ and $\partial H / \partial y_{i}$ are real, equating to zero the derivatives of $H$ with regard to $\bar{z}_{i}$ gives an equation equivalent to the two

* $A$ new method in boundary problems for differential equations, vol. 18, pp. 495-496. 
obtained by equating to zero the derivatives $\partial H / \partial x_{i}$ and $\partial H / \partial y_{i}$. In other words, each of the $n$ equations

$$
\sum_{j}\left(a_{i j}-\lambda b_{i j}\right) z_{j}=0 \quad(i=1,2, \cdots, n)
$$

is equivalent to the 2 linear homogeneous equations obtained by deriving with respect to $x_{i}$ and $y_{i}$. The set (7) contains $n$ equations in $n$ complex variables and the others form a set of $2 n$ equations in $2 n$ real variables.

By a theorem analogous to I (XV) each solution-multiplicity of (7) is unity and hence the solutions for the various $\lambda$ 's can be written down as linearly independent. The solution for the conjugate equations can likewise be written down, each being conjugate to one of the other set. One of these solutions $z, \bar{z}$ when normalized to satisfy the relation $B(z, \bar{z})=1$ (which may also be considered as obtained from (5) by differentiation with regard to $\lambda$ ), must furnish the minimum for $A(z, \bar{z})$. Since these give to $A$ the values $\left(3^{\prime}\right) \lambda_{1} \leqq \cdots \leqq \lambda_{n}$, the minimum must be furnished by $Z^{(1)}, \bar{Z}^{(1)}$ which gives to $A$ the value $\lambda_{1}$; more specific discussion is reserved for the next section.

To determine the minimum under the added orthogonal condition

$$
B\left(Z^{(1)}, \bar{z}\right)=0 \quad\left(\text { or the conjugate } B\left(z, \bar{Z}^{(1)}\right)=0\right),
$$

we set up as the expression to be minimized

$$
A(z, \bar{z})-\lambda[B(z, \bar{z})-1]-2 \mu\left[B\left(Z^{(1)}, \bar{z}\right)\right],
$$

and differentiate with regard to the $\bar{z}$ 's, $\lambda$ and $\mu$, obtaining $n+1$ linear homogeneous equations in the $z$ 's and $\mu$,

$$
\sum_{j}\left(a_{i j}-\lambda b_{i j}\right) z_{j}-\mu \sum_{j} b_{i j} z_{j}^{(1)}=0,
$$

together with (8) and the quadratic relation of (5). Each of the derived equations (9) in $z$ is equivalent to two equations, both containing $x$ 's and $y$ 's; and it is easily shown that these two are the derived equations of those which would be set up from one of the equations (7) by separation into real and imaginary parts. In order that these linear equations in $z_{i}$ and $\mu$ have a solution, the necessary and sufficient condition is the vanishing of the determinant formed by bordering $\left|a_{i j}-\lambda b_{i j}\right|$ with one row and one column only (cf. (10)). This polynomial in $\lambda$, which is real 
because the terms symmetrical with the main diagonal are conjugate, is of the $(n-1)$ th degree and we can readily show that the roots are $\lambda_{2}, \cdots, \lambda_{n}$. For, if we set $\lambda=\lambda_{i}(i=2, \cdots, n)$ and multiply the first $n$ rows respectively by $\bar{z}_{1}^{(i)}, \ldots, \bar{z}_{n}^{(i)}$ and add, we have zero for each column. This is true for the first $n$ columns because the solution $Z^{(i)}$ is so defined, while for the last $((n+1)$ th $)$ column it follows from the orthogonality relation (8).

To show that $\mu$ is zero we multiply the equations (9) by $\bar{z}_{1}^{(1)}, \ldots, \bar{z}_{n}^{(1)}$ respectively and add, giving for the first $n$ columns

$$
z_{j} \sum_{i}\left(a_{i j}-\lambda b_{i j}\right) z_{i}^{(1)} \quad(j=1, \cdots, n)
$$

the coefficients of $z_{j}$ being conjugates of the left hand sides of (7) and hence zero. For the other part we have on addition $\mu B\left(z^{(1)}, \bar{z}^{(1)}\right)$, which must then be zero from the equations (9); and from (5) it follows that $\mu=0$. Equations (9) reduce to (7) but since for solutions of such equations we have, from $\left(3^{\prime}\right), A\left(z_{i}, \bar{z}_{i}\right)=\lambda_{i} B\left(z_{i}, \bar{z}_{i}\right)$, the minimum process picks out the solution $Z^{(2)}, \bar{Z}^{(2)}$ which when normalized to satisfy (5) furnishes to $A$ the value $\lambda_{2}$.

Proceeding in the same manner we can show successive minima to exist and that they are furnished by solutions of the equations (7) for $\lambda=\lambda_{3}, \cdots$. The expression to be used in the last case when the additional orthogonal conditions

are imposed is

$$
\sum_{i j} b_{i j} z_{i}^{(2)} \bar{z}_{j}=0, \quad \cdots, \quad \sum_{i j} b_{i j} z_{i}^{(n-1)} \bar{z}_{j}=0
$$

$$
A(z, \bar{z})-\lambda B(z, \bar{z})-2 \mu_{1} B\left(Z^{(1)}, \bar{z}\right)-\cdots-2 \mu_{n-1} B\left(Z^{(n-1)}, \bar{z}\right)
$$

and the determinant of the linear equations is

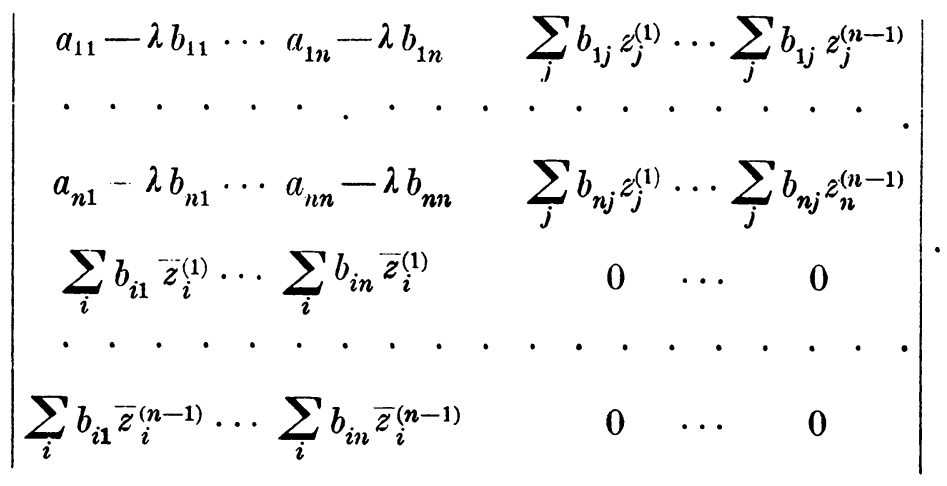


The polynomial in $\lambda$ is real and of the first degree and since on setting $\lambda=\lambda_{n}$ and multiplying the first $n$ rows by $\bar{z}_{1}^{(n)}, \ldots, \bar{z}_{n}^{(n)}$ respectively and adding, the sum of each column is zero, it follows that this polynomial has $\lambda_{n}$ for zero. To show that $\mu_{i}=0$, we multiply the first $n$ linear equations corresponding to the matrix (10) by $\bar{z}_{1}^{(i)}, \ldots, \bar{z}_{n}^{(i)}$ and on addition note that the coefficients of the $z_{j}$ in the first $n$ columns are the conjugates of the left hand sides of (7) and hence are by hypothesis zero for $i=1, \cdots, n$. On account of the orthogonal conditions imposed, the sums of all the other columns so obtained will also be zero, except that the $(n+i)$ th gives $\mu_{i} B\left(z^{(i)}, \bar{z}^{(i)}\right)$ and since this must also be zero, it follows that $\mu_{i}=0$. The first $n$ equations reduce then to (7) and, by ( $\left.3^{\prime}\right)$, it follows that $A$ has a minimum (and only) value $\lambda_{n}$.

When neither of the hermitian forms is definite some or all of the characteristic numbers may be complex. The complex $\lambda$ 's occur in conjugate imaginary pairs and the corresponding solutions will be conjugate imaginary. In this case by proceeding formally and imposing the orthogonal conditions which naturally arise the problem may eventually be reduced to one which has real significance.*

As a typical example where both forms are indefinite and where solutions of the derived equations arise in the formal solution of the minimum problem, let us consider the problem of $I(\S 6)$ connected with the matrix (33) and in order that it may have a meaning as a minimum problem let us consider the form as quadratic and not bilinear.t If the coefficients of $A$ and $B$ are real the $\lambda$ 's here will be real but in similar problems some might well be complex, with another set conjugate to them.

For this problem the linear equations obtained by differentiating with regard to the $x$ 's the expression $A(x, x)-\lambda[B(x, x)-c]$ have solutions which make $B(x, x)=0$, so that in order to satisfy the quadratic equation also we must modify by setting $c=0$ before proceeding. The next step formally is to minimize

$$
A(x, x)-\lambda\left[B(x, x)-c_{1}\right]-2 \mu\left[B\left(X^{(1)}, x\right)-c_{2}\right],
$$

where $X^{(1)}$ is the formal solution of the modified first problem. This gives rise to the linear equations which we have called derived equations (the

* For example, if

$$
A=2 x_{1} x_{2}+2 x_{3}^{2}-2 x_{4}^{2}, \quad B=x_{1}^{2}-x_{2}^{2}+x_{3}^{2}+x_{4}^{2},
$$

the $\lambda$ 's are $i,-i, 1,-2$, the formal orthogonal conditions corresponding to $i$ and $-i$ are $x_{1}=0, x_{2}=0$ and since after these conditions are imposed, $B$ is definite, the resulting problem may be solved in the usual manner.

$\dagger$ A part of the formal work can be carried out even with bilinear forms by differentiating both with regard to $x$ 's and $y$ 's. 
multiplier $\mu$ can be absorbed into the solution $X^{(1)}$ ). In a manner similar to that used above with hermitian forms it can be shown that the polynomial in $\lambda$ resulting from the determinant formed from the four linear equations in $x_{1}, x_{z}, x_{3}, \mu$ is of the second degree and that $\lambda_{1}$ is a (double) root. The solution $X^{(2)}$ of the derived equations can be made to satisfy the quadratic condition $B\left(X^{(2)}, X^{(2)}\right)=1$ while $B\left(X^{(1)}, X^{(2)}\right)$ is zero no matter what solution is chosen for $X^{(2)}$. Since $B\left(X^{(1)}, X^{(1)}\right)=0$, the argument that $\mu=0$, given in connection with the equations (9), is no longer valid; in fact $\mu$ must be different from zero. For, since if $\mu=0$, equations (9) reduce to (7), which here have by hypothesis one solution only, it would follow that $X^{(2)}=X^{(1)}$ and this would lead nowhere.

Proceeding to the final stage we set up the expression

$$
A(x, x)-\lambda\left[B(x, x)-c_{1}\right]-2 \mu\left[B\left(X^{(1)}, x\right)-c_{2}\right]-2 \nu\left[B\left(X^{(2)}, x\right)-c_{3}\right]
$$

and derive with regard to $x_{1}, x_{2}, x_{3}, \mu, \nu, \lambda$. The five linear equations furnish a determinant which on equating to zero gives a polynomial of first degree for $\lambda$. It may be shown that $\lambda=\lambda_{1}$ is the root and $X^{(8)}$ a solution. By a slight extension of the methods in I (§6) it may be shown that there are in these linear equations enough arbitrary constants and solutions capable of adjustment to make $B\left(X^{(3)}, X^{(3)}\right)=0, B\left(X^{(2)}, X^{(8)}\right)=0$ while $B\left(X^{(1)}, X^{(3)}\right)=B\left(X^{(2)}, X^{(2)}\right)$.

3. A NEW NECESSARY CONDITION FOR RELATIVE EXTREMA IN QUADRATIC AND HERMITIAN FORMS. SUFFICIENT CONDITIONS

In determining in $\S 2$ the minimum of the hermitian form $A(z, \bar{z})$ under the quadratic condition that the positive definite hermitian form $B(z, \bar{z})$ be unity and under the $m$ linear conditions

$$
B\left(Z^{(1)}, \bar{z}\right)=0, \quad \cdots \quad B\left(Z^{(m)}, \bar{z}\right)=0,
$$

we were led to the consideration of a determinant $D(\lambda)$ made up of the determinant $\left|a_{i j}-\lambda b_{i j}\right|$ bordered by $m$ rows and columns (cf. (10) or (13)). This determinant is of degree $n-m$ in $\lambda$ and, as the author has shown in another paper*, the zeros of such a polynomial are all real. In $\S 2$, it was shown that these zeros are actually $\lambda_{m+1}, \ldots, \lambda_{n}$, where $\lambda_{1} \leqq \lambda_{2} \leqq \cdots \leqq \lambda_{n}$ are the zeros of the unbordered determinant $\left|a_{i j}-\lambda b_{i j}\right|$. For each of these $n-m$ values $\lambda_{k}$, the linear equations corresponding to

\footnotetext{
* On the reality of the zeros of a $\lambda$-determinant, Bulletin of the American Mathematical Society, vol. 29, p. 467. See also Hilton, Linear Substitutions, for a somewhat less general theorem.
} 
this determinant may be solved and the solutions normalized, giving $Z^{(m+1)}, \cdots, Z^{(n)}$, while $\bar{Z}^{(m+1)}, \cdots, \bar{Z}^{(n)}$ are the solutions of the conjugate equations. One of these must furnish the minimum sought and since as in $\left(3^{\prime}\right)$ we have

$$
A\left(Z^{(k)}, \bar{Z}^{(k)}\right)=\lambda^{(k)} B\left(Z^{(k)}, \bar{Z}^{(k)}\right),
$$

it is necessary that the minimum of $A(z, \bar{z})$ be $\lambda_{m+1}$, which is the smallest of the $n-m$ possibilities. It is evident at this point that there must be some necessary condition which picks out from the $n-m$ solutions available the particular one, $Z^{(m+1)}, \bar{Z}^{(m+1)}$, to furnish the minimum.

Let us consider the minimum of the hermitian form $A(z, \bar{z})-\lambda_{k} B(z, \bar{z})$ subject to the conditions (11). The corresponding determinant $D\left(\lambda_{k}\right) \equiv$

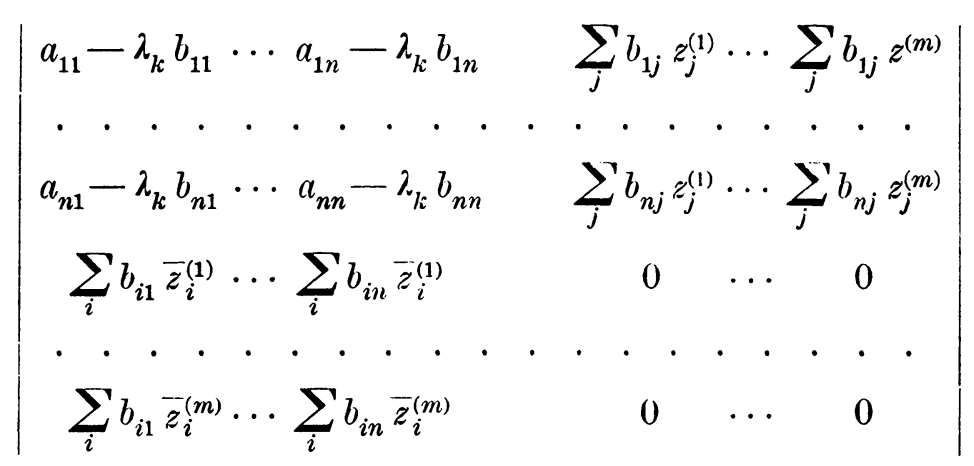

may be obtained by setting $\lambda=\lambda_{k}$ in $D(\lambda)$. From the discussion of the previous sections and (12) it is clear that when $k=m+1$ the minimum of $A-\lambda_{k} B$ is zero, while for larger values of $\lambda_{k}$, it is negative.

On the other hand, as has been shown in the preceding sections, for values of the variables which in addition to the conditions (11) are normalized by the relation $z_{1} \bar{z}_{1}+\cdots+z_{n} \bar{z}_{n}=1$, the minimum of $A-\lambda_{k} B$ is one of the $n-m$ real* zeros of the determinant in $\mu$

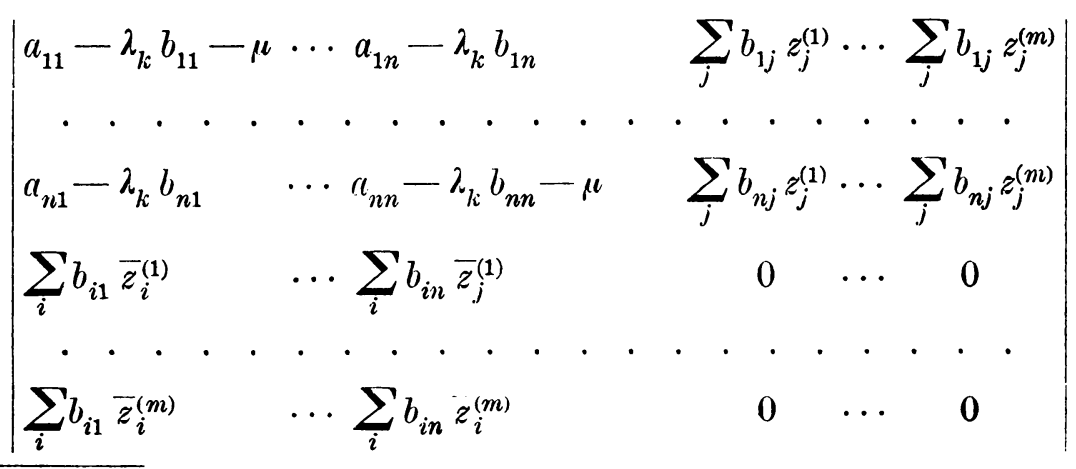

* Loc. cit. 
obtained from $D\left(\lambda_{k}\right)$ by adding $-\mu$ to each of the first $n$ terms in the main diagonal. The minimum will be the smallest of these $n-m$ zeros and hence for $k=m+1$ none of these can be negative, while for $k=m+2, \cdots, n$ some may be negative. But to ensure that a polynomial in $\mu$, all of whose roots are real, have no negative root, it is necessary and sufficient that there be no permanences of sign in the sequence of its coefficients.

A simple way to obtain the coefficients of the polynomial (14) is to break it up into the sum of $2^{n}$ non-identically vanishing determinants such that for each of them any particular column consists either of terms in $\mu$ only or else of elements of (14) with $\mu$ deleted. Let us denote by $\sigma_{p}$ the coefficient of $\mu^{p}(0 \leqq p \leqq n-m)$. Then it is easily proved that

$$
\sigma_{p}=\sum D_{r_{1} r_{2} \ldots r_{p}}, \quad r_{1}, r_{2}, \cdots, r_{p} \leqq n,
$$

where $D_{r_{1} r_{3} \ldots r_{p}}$ is the minor of (13) obtained by the deletion of the $r_{1} r_{2} \ldots r_{p}$ rows and the same columns and where the summation extends over all possible minors obtained by such deletion. In order that the polynomial in $\mu$ have no negative root, it is necessary and sufficient that there be no permanences of sign in the sequence $\sigma_{0}=0, \sigma_{1}, \ldots, \sigma_{n-m}$, and this is true even when $\sigma_{1}=0, \ldots, \sigma_{q-1}=0$ and $\lambda_{k}$ is a $q$-tuple root. This constitutes the new necessary condition for an extremum.

The results may now be expressed as

THEOREM IV. In order that the hermitian form $A(z, \bar{z})$ have a minimum subject to the conditions that the positive definite hermitian form $B(z, \bar{z})$ be unity and that the linear conditions (11) be satisfied where $Z^{(1)}, \ldots, Z^{(m)}$; $\bar{Z}^{(1)}, \ldots, \bar{Z}^{(m)}$ are the solutions of the sets of linear equations

$$
\sum_{j}\left(a_{i j}-\lambda b_{i j}\right) z_{j}=0, \quad \sum_{i}\left(a_{i j}-\lambda b_{i j}\right) \bar{z}_{i}=0,
$$

respectively, corresponding to the $m$ smallest of the zeros $\lambda_{1} \leqq \lambda_{2} \leqq \cdots \leqq \lambda_{n}$ of the determinant $\left|a_{i j}-\lambda b_{i j}\right|$, the necessary and sufficient conditions are

(i) that the sets of values $Z^{(k)} \equiv\left(z_{1}^{(k)}, \ldots, z_{n}^{(k)}\right), \bar{Z}^{(k)} \equiv\left(\bar{z}_{1}^{(k)}, \ldots, \bar{z}_{n}^{(k)}\right)$ furnishing the minimum be solutions respectively of the sets of equations (16);

(ii) that $\lambda_{k}$ be a zero of the determinant (13) and that for the particular root $\lambda_{k}$ of the determinant $\left|a_{i j}-\lambda b_{i j}\right|$ which furnishes the minimum, the sequence of sums $\sigma_{0}, \sigma_{1}, \cdots, \sigma_{n-m}(15)$ made up from (13) have no permanence of sign.

A special case of this theorem is that relating to real quadratic forms. 
When no linear conditions (11) are imposed, the $\sigma_{i}$ represent sums of principal minors of the determinant $\left|a_{i j}-\lambda_{k} b_{i j}\right|$.

It is not difficult to write down from considerations of symmetry the corresponding theorem for maxima.

Brown UnIVersity,

Providence, R. I.

\section{ERRATA, VOLUME 25}

J. F. RITT, Permutable rational functions.

Page 399, second line from bottom (footnote), for "exists" read "exist."

Page 402, line 21, for "see the" read "see how the."

Norbert Wrener, Discontinuous boundary conditions and the Dirichlet problem.

Page 313, line 14, the exponent of $(P Q)$ should be $2-n$, not $n-2$.

Page 314, line 1, same correction.

Page 314, line 3, for " $c a^{n-2}$ " read " $c a^{2-n} "$. 